\title{
Development and Full Validation of a Bioanalytical Method for Quantifying Letermovir in Human Plasma Using Ultra-Performance Liquid Chromatography Coupled with Mass Spectrometry
}

\author{
Tassadit Belabbas, ${ }^{a}$ Takaaki Yamada ${ }^{b}$ Yuichi Tsuchiya,${ }^{b}$ Kimitaka Suetsugu, ${ }^{b}$ \\ Nobuaki Egashira, ${ }^{a, b}$ and Ichiro Ieiri* ${ }^{*, a, b}$ \\ ${ }^{a}$ Department of Clinical Pharmacology and Biopharmaceutics, Graduate School of Pharmaceutical Sciences, \\ Kyushu University; 3-1-1 Maidashi, Higashi-ku, Fukuoka 812-8582, Japan: and ${ }^{b}$ Department of Pharmacy, Kyushu \\ University Hospital; 3-1-1 Maidashi, Higashi-ku, Fukuoka 812-8582, Japan. \\ Received February 15, 2021; accepted March 30, 2021
}

\begin{abstract}
With the aim of studying the pharmacokinetics of letermovir, which is a newly developed antiviral agent for human cytomegalovirus, a rapid and simple ultra-performance liquid chromatography coupled with mass spectrometry (UPLC/MS) method was developed and validated for the quantification of letermovir in human plasma. Separation was performed in reverse phase mode using an ACQUITY UPLC BEH C18 column (130 ̊, $1.7 \mu \mathrm{m}, 2.1 \times 50 \mathrm{~mm}$ ) at a flow rate of $0.3 \mathrm{~mL} / \mathrm{min}, 10 \mathrm{mM}$ ammonium acetate- $0.1 \%$ formic acid solution as $\mathrm{mo}$ bile phase $\mathrm{A}$, and acetonitrile as mobile phase $\mathrm{B}$ with a gradient elution. The method was validated over a linear range of $10-1000 \mathrm{ng} / \mathrm{mL}$ with a coefficient of determination $\left(R^{2}\right)>0.99$ using weighted linear regression analysis. The intra- and inter-assay accuracy (nominal\%) and precision (relative standard deviation\%) were within \pm 15 and $\leq 15 \%$, respectively. The specificity, recovery, matrix effect, stability, and dilution integrity of this method were also within acceptable limits. This method could be useful in studying the pharmacokinetics and pharmacodynamics, as well as performing the therapeutic drug monitoring of letermovir.
\end{abstract}

Key words letermovir; bioanalytical method development; validation; ultra-performance liquid chromatography coupled with mass spectrometry; human plasma

\section{Introduction}

Letermovir is a newly developed antiviral agent indicated in the prophylaxis of human cytomegalovirus (CMV) infections in adult recipients of allogeneic hematopoietic stem cell transplants (HSCT) ${ }^{1-3)}$ Unlike other antiviral agents such as ganciclovir, foscarnet, and cidofovir, which target the viral DNA polymerase, ${ }^{4)}$ letermovir inhibits viral DNA cleavage by targeting the CMV terminase complex, pUL56. ${ }^{5)}$ It is commercially available in both intravenous and oral formulations with a recommended therapeutic dose of $480 \mathrm{mg}$ once daily. $\left.{ }^{6}\right)$

Recently, several studies have reported various cases of CMV resistance during letermovir prophylaxis in HSCT recipients. ${ }^{7-9}$ ) Furthermore, letermovir was found to increase tacrolimus exposure by inhibiting CYP $3 \mathrm{~A} 4^{10,11)}$ and decrease voriconazole exposure in healthy recipients secondary to CYP2C9/19 induction. ${ }^{12)}$ It has also been found that coadministration with cyclosporine significantly decreases letermovir clearance, leading to an increase in letermovir exposure caused by the inhibitory effect of cyclosporine on organic anion transporting polypeptide (OATP) $1 \mathrm{~B} 1 / 3$ transporters; OATP1B1/3 is a hepatic metabolizer of letermovir. ${ }^{11}$

In light of these findings, further studies for assessing the pharmacokinetics (PK) and pharmacodynamics (PD) are required. However, although multiple clinical studies ${ }^{3,10,11,13)}$ mention the use of LC/MS methods for quantifying letermovir in human plasma, there is no information regarding the full method, including its validation in the available literature. More recently, a new method for the quantification of letermovir in human serum using HPLC combined with diode array detector has been published. ${ }^{14)}$ However, to date, no LC/MS method has been described in human plasma. Thus, the main purpose of this work was to develop and validate an ultraperformance liquid chromatography (UPLC)/MS method for quantifying letermovir in human plasma, which is suitable for clinical studies as well as for therapeutic drug monitoring.

\section{Experimental}

Chemicals and Reagents The reference standard letermovir ( $\geq 98 \%$ purity) was purchased from Cayman Chemical (Ann Arbor, Michigan, U.S.A.). The chemical structure of letermovir is presented in Fig. $1 .^{5)}$ Roscovitine, which was used as the internal standard (IS), was purchased from Wako Pure Chemical Corporation (Osaka, Japan). Methanol (MeOH), acetonitrile (ACN), dimethylsulfoxide (DMSO, 99\% purity), and formic acid (98\% purity) were supplied by Wako Pure Chemical Corporation. Methanol and acetonitrile were both<smiles>COc1cccc(N2CCN(C3=Nc4c(F)cccc4[C@@H](CC(=O)O)N3c3cc(C(F)(F)F)ccc3OC)CC2)c1</smiles>

Fig. 1. Chemical Structure of Letermovir $\left(\mathrm{C}_{29} \mathrm{H}_{28} \mathrm{~F}_{4} \mathrm{~N}_{4} \mathrm{O}_{4}\right)^{5)}$ 
LC/MS grade. Ammonium formate $(1 \mathrm{~mol} / \mathrm{L})$ and ammonium acetate $(10 \mathrm{~mol} / \mathrm{L})$ were purchased from Nacalai Tesque, Inc. (Kyoto, Japan). Milli-Q water (of $18.2 \mathrm{~m} \Omega$ ) was obtained from an in-house purification system, Elga LabWater PURELAB ${ }^{\circledR}$ flex (Elga LabWater, High Wycombe, U.K.). Human blank plasma K2 dipotassium ethylenediaminetetraacetic acid (EDTA) was purchased from Cosmo Bio Co., Ltd. (Tokyo, Japan). Letermovir reference standard and human blank plasma were stored at $-20^{\circ} \mathrm{C}$ prior to use.

Equipment The LC system consisted of an ACQUITY UPLC $^{\circledR}$ system, equipped with an autosampler, a binary solvent manager, and an online degasser. Analyte separation was performed in reverse phase mode with an ACQUITY UPLC ${ }^{\circledR}$ BEH C18 column $(130 \AA, 1.7 \mu \mathrm{m}, 2.1 \times 50 \mathrm{~mm})$ preceded by Waters ACQUITY BEH C18 Van-Guard pre-column and detected using Waters ACQUITY ${ }^{\circledR}$ QDa detector. The chromatography system, column, and mass spectrometer were purchased from the Waters Corporation (Milford, MA, U.S.A.). Data manipulation was performed using the Empower 3 software (Waters Corporation).

Method Development During method development, a large number of chromatographic and mass spectrophotometric parameters were screened and optimized by using a screening protocol, in order to develop a fast and robust bioanalytical method for quantifying letermovir in human plasma.

\section{MS Conditions}

Detection of letermovir and IS, based on the peaks' mass to charge $(\mathrm{m} / \mathrm{z})$ ratio, was carried out using Waters ACQUITY ${ }^{\circledR}$ QDa detector through electrospray ionization (ESI) operating in a positive ion mode. The cone and capillary voltages were $15 \mathrm{~V}$ and $0.8 \mathrm{kV}$, respectively. The probe temperature was set to $600{ }^{\circ} \mathrm{C}$, and the sampling rate was set to 10 points/s.

\section{Chromatographic Conditions}

Chromatographic separation was performed in reverse phase mode with an ACQUITY UPLC BEH C18 column maintained at $50{ }^{\circ} \mathrm{C}$. The mobile phase was delivered at a flow rate of $0.3 \mathrm{~mL} / \mathrm{min}$ through gradient elution and consisted of $10 \mathrm{mM}$ ammonium acetate in pure water with $0.1 \%$ formic acid added for $\mathrm{pH}$ adjustment (aqueous mobile phase A) and acetonitrile (organic mobile phase B). The total analytical run time for each injection was $6.1 \mathrm{~min}$, including $2 \mathrm{~min}$ of re-equilibration.

The initial gradient conditions started with $10 \%$ mobile phase B which was maintained for $0.5 \mathrm{~min}$. Mobile phase B was gradually increased to $40 \%$ over $1 \mathrm{~min}$ and further increased to $90 \%$ for $1.4 \mathrm{~min}$. These conditions were held for $1.1 \mathrm{~min}$, returned to initial conditions over $0.1 \mathrm{~min}$, and maintained for $2 \mathrm{~min}$. Optimization of chromatographic conditions involved the consecutive screening of the following parameters: buffer, $\mathrm{pH}$ of the mobile phase, organic solvent, gradient elution, flow rate, column temperature, and injection volume. The initial method conditions for the first screening were selected according to letermovir physicochemical properties $\left(\mathrm{p} K_{\mathrm{a}}\right.$ values, molecular weight, solubility, and stability). The conditions offering the best retention and separation were then selected.

Preparation of the Stock Solution, Calibration Standards, and Quality Control (QC) Working Solutions

Stock solution was prepared at $1 \mathrm{mg} / \mathrm{mL}$ by dissolving $5 \mathrm{mg}$ of letermovir standard in DMSO. The stock solution was further diluted in an appropriate volume of methanol-water $(1: 1$, $\mathrm{v} / \mathrm{v})$ to prepare a second stock solution at $100 \mu \mathrm{g} / \mathrm{mL}$. From the second stock solution, two individual working solutions (calibration standard working solution and QC working solutions) were freshly prepared in methanol-water $(1: 1, \mathrm{v} / \mathrm{v})$ at 10 and $7.5 \mu \mathrm{g} / \mathrm{mL}$, respectively. Serial dilution with methanol-water $(1: 1, \mathrm{v} / \mathrm{v})$ was then performed in order to obtain six calibration standard working solutions and three QC working solutions. The IS working solution was prepared at $5 \mu \mathrm{g} / \mathrm{mL}$ by diluting the corresponding stock solution in methanol-water $(1: 1, \mathrm{v} / \mathrm{v})$.

\section{Calibration Standards, and QC Samples}

Calibration standard working solutions were diluted 10-fold with human blank plasma $(1: 9, \mathrm{v} / \mathrm{v})$ to obtain six calibration standards at 10, 100, 200,400, 800, and $1000 \mathrm{ng} / \mathrm{mL}$. QC working solutions were also diluted 10 -fold with human blank plasma to obtain QCs at three different concentration levels: i.e., low-, mid-, and high-: levels of 30,500 , and $750 \mathrm{ng} / \mathrm{mL}$, respectively.

Samples Pre-treatment

Ten microliters of the IS working solution were added to $1.5 \mathrm{~mL}$ centrifuge polyethylene tubes containing $100 \mu \mathrm{L}$ of the corresponding calibration standard or QC diluted in human blank plasma. Protein precipitation was induced by adding $400 \mu \mathrm{L}$ of methanol to all the samples. The tubes were vortexed for $1 \mathrm{~min}$ and centrifuged at $13000 \times \boldsymbol{g}$ for $5 \mathrm{~min}$. The supernatant was diluted with an appropriate volume of mobile phase $(1: 1, \mathrm{v} / \mathrm{v})$, and $2 \mu \mathrm{L}$ was injected into the LC/MS system.

Method Validation Full validation of the method in human plasma was performed in compliance with the U.S. Food and Drug Administration (FDA) guidelines on bioanalytical method development and validation. ${ }^{15)}$ The validation included the following parameters: linearity and calibration curve, carry over, selectivity, intra-, and inter-assay accuracy and precision, recovery and matrix effect, dilution linearity, and stability. ${ }^{16)}$

Linearity and Calibration Curve

Calibration standards were prepared at six concentrations over a range of $10-1000 \mathrm{ng} / \mathrm{mL}$. Six individually prepared replicates at each concentration, including a blank and a zero calibrator standard (blank plasma spiked with IS), were analyzed within six different runs. In order to confirm the concentration-response relationship, calibration curves were plotted by fitting the peak response ( $y$-axis), defined as the peak-area ratio of letermovir to IS, against the nominal concentration of the calibration standard ( $x$-axis).

Concentrations were calculated using unweighted and weighted linear least-squares regression analysis with the weighting factors: $1,1 / x$, and $1 / x^{2}$, where $x$ is the concentration of letermovir. The choice of the weighting factor was based on the relationship between the standard deviation and variance of the LC/MS response and the analyte concentration. ${ }^{17)}$

The coefficient of determination $\left(R^{2}\right)$ should be $\geq 0.99$. Accuracy (nominal\%) and precision (relative standard deviation (RSD) \%) at each concentration should be $\pm 15 \%$ and $\leq 15 \%$, respectively, except at the lower limit of quantification (LLOQ), where the calculated concentration should be $\pm 20 \%$ of the nominal concentration and RSD $\leq 20 \%$.

\section{Carryover}

Carryover was evaluated by analyzing a human blank plasma following the injection of an upper limit of quantification (ULOQ), the highest concentration calibration standard, in five different runs. Carryover should not exceed $20 \%$ of the 
LLOQ.

\section{Selectivity}

Selectivity was defined as the absence of substances interfering with the analyte and IS at the same retention time and was evaluated by analyzing six different human plasma samples prepared at LLOQ $(10 \mathrm{ng} / \mathrm{mL})$ and IS at $500 \mathrm{ng} / \mathrm{mL}$. The human plasma samples should be free of interferences with the elution of the analyte and IS. If any peak is observed at the retention time of the analyte and/or IS, the response should be $\leq 20 \%$ of the LLOQ response and $\leq 5 \%$ of the response of the IS.

Accuracy and Precision

Accuracy and precision were evaluated at LLOQ level, and with QCs prepared at three different concentrations. Repeatability or intra-assay accuracy, defined as nominal $\%$, and precision (RSD\%), were determined by analyzing five individually prepared replicates at each concentration within the same run and five injections of one replicate within another run to evaluate injection repeatability.

Inter-assay accuracy and precision were obtained by analyzing five individually prepared replicates at each concentration within five different days. Accuracy at each concentration level should be within $\pm 15 \%$, and precision should be $\leq 15 \%$, except at the LLOQ where accuracy and precision should be \pm 20 and $\leq 20 \%$, respectively.

Matrix Effect and \% Recovery

QCs at low, mid, and high levels were prepared in six different human plasma samples. The matrix factor was evaluated by comparing the peak area of the QC prepared in human plasma with the peak area of the QC prepared in methanolwater $(1: 1, \mathrm{v} / \mathrm{v})$.

Recovery was evaluated by comparing the peak area of the extracted QC prepared at three levels of concentration with the peak area of the extracted human plasma sample spiked with letermovir and IS at the same concentration. Precision expressed by the RSD\% for both the matrix effect and recovery should not exceed $15 \%$.

Dilution Linearity

Plasma dilution in human blank plasma was assessed. Five individually prepared replicates of QCs at 10 times $(10 \mu \mathrm{g} / \mathrm{mL})$ the ULOQ were analyzed. The samples were diluted 10 times with human blank plasma to obtain samples of $1000 \mathrm{ng} / \mathrm{mL}$. The mean accuracy at each level of concentration should be within $\pm 15 \%$, and the precision expressed in $\mathrm{RSD} \%$ should be $\leq 15 \%$.

\section{Stability}

The stability of the stock solution was assessed by analyzing three individually prepared replicates in methanol-water $(1: 1, \mathrm{v} / \mathrm{v})$ at $1 \mu \mathrm{g} / \mathrm{mL}$, after storage at room temperature (r.t., $24^{\circ} \mathrm{C}$ ) for $6 \mathrm{~h}$, and after storage at $-20^{\circ} \mathrm{C}$ for 6 months and 12 months. The stability of letermovir in human blank plasma was determined using five individually prepared replicates of QCs at three concentration levels. The following stability conditions were assessed: short-term stability $(24 \mathrm{~h}$ at r.t. and at $-20^{\circ} \mathrm{C}$ ), post-preparation $\left(48 \mathrm{~h}\right.$ at $\left.-20^{\circ} \mathrm{C}\right)$, auto-sampler $\left(24 \mathrm{~h}\right.$ at $\left.10^{\circ} \mathrm{C}\right)$, freeze thaw (three cycles, $-20^{\circ} \mathrm{C} / \mathrm{r}$.t., and $24 \mathrm{~h}$ between cycles), and long-term stability $\left(10 \mathrm{~d}\right.$ at $-20^{\circ} \mathrm{C}$, and $30 \mathrm{~d}$ at -20 and $-80^{\circ} \mathrm{C}$ ). Letermovir was considered stable under the different storage conditions if the accuracy (nominal $\%$ ) and precision (RSD $\%$ ) were within \pm 15 and $\leq 15 \%$, respectively.

\section{Results and Discussion \\ Method Development}

Mass Spectrometry

The ionization conditions used in the full mass scan permitted the detection of prominent peaks for letermovir and IS with $\mathrm{m} / \mathrm{z}$ of 573.30 and 355.31, respectively. Single chromatograms of letermovir were obtained by setting the QDa mass spectrometer to single $\mathrm{m} / \mathrm{z}$ values previously obtained through a selected ion recording (SIR). The results of the full mass scan and SIR showed that no further optimization was required. Thus, initial ESI-MS was selected for optimizing the chromatographic conditions.

LC

The chromatographic method was optimized by changing various parameters such as $\mathrm{pH}$ of the buffer and organic solvent. Best separation, elution, and tailing were obtained with $10 \mathrm{mM}$ ammonium acetate in pure water and $0.1 \%$ formic acid to obtain a pH of 5.2 (mobile phase A) and acetonitrile (mobile phase B). Furthermore, to achieve a capacity factor $\geq 2$, the column temperature was maintained at $50{ }^{\circ} \mathrm{C}$ and acetonitrile was gradually increased from 10 to $90 \%$, over $2.9 \mathrm{~min}$.

\section{Method Validation}

\section{Linearity and Calibration Curve}

The equations of linear regression and coefficients of determination $R^{2}$ are described in Table 1. The concentrationresponse relationship was best described with a $1 / x^{2}$ weighting factor. $R^{2}$ of six replicates was $>0.99$, and the accuracy of all six calibration points was within $\pm 15 \%$ of the nominal concentration. Thus, the calibration curve was validated over a range of $10-1000 \mathrm{ng} / \mathrm{mL}$.

The choice of the upper limit of $1000 \mathrm{ng} / \mathrm{mL}$ was based on the results of previous clinical trials. ${ }^{3,11)}$ The predicted median minimal concentration of letermovir in Asian patients after oral administration of $480 \mathrm{mg}$, and after oral administration of $240 \mathrm{mg}$ with cyclosporine exposure were $506.4 \mathrm{ng} / \mathrm{mL}$ with a $90 \%$ prediction interval of $118.4-1628$, and $1200 \mathrm{ng} / \mathrm{mL}$ with a $90 \%$ prediction interval of 346.9-3154, respectively. ${ }^{3)}$ Furthermore, according to a study conducted by Isberner et al., the median trough concentration of letermovir after oral administration of 240 or $480 \mathrm{mg}$ in patients including children was $2603 \mathrm{ng} / \mathrm{mL}$ (range $175-12281 \mathrm{ng} / \mathrm{mL}$ ), and two samples were less than $100 \mathrm{ng} / \mathrm{mL}^{14)}$ These results suggest that letermovir

Table 1. Calibration Curve and Regression Parameters Results Obtained with Unweighted and Weighted Linear Regression Analysis

\begin{tabular}{ccc}
\hline \hline Weighting factor & Mean equation for regression line & Mean coefficient of determination $\left(R^{2}\right)$ \\
\hline 1 & $y=0.000873 x+0.002119$ & 0.9995 \\
$1 / x$ & $y=0.000879 x-0.000336$ & 0.9997 \\
$1 / x^{2}$ & $y=0.000882 x-0.000484$ & 0.9995 \\
\hline
\end{tabular}

a) Regression parameters obtained with calibration curves of six standards in six analytical runs. $b$ ) Mean relative error (RE)\%: ((obtained concentration - nominal concentration)/nominal concentration) $\times 100$ 
concentrations exhibit a large variability in clinical setting. In this study, a lower ULOQ than that used in a previous study $(5000 \mathrm{ng} / \mathrm{mL})^{14)}$ was chosen to improve the accuracy of our calibration curve, while a dilution integrity of 10 -fold was assessed in order to account for concentrations above $1000 \mathrm{ng} / \mathrm{mL}$.

\section{Carryover}

No response was detected in the five replicates of human blank plasma samples processed after a ULOQ sample at the retention time of letermovir and IS. Thus, no carry-over effect was observed.
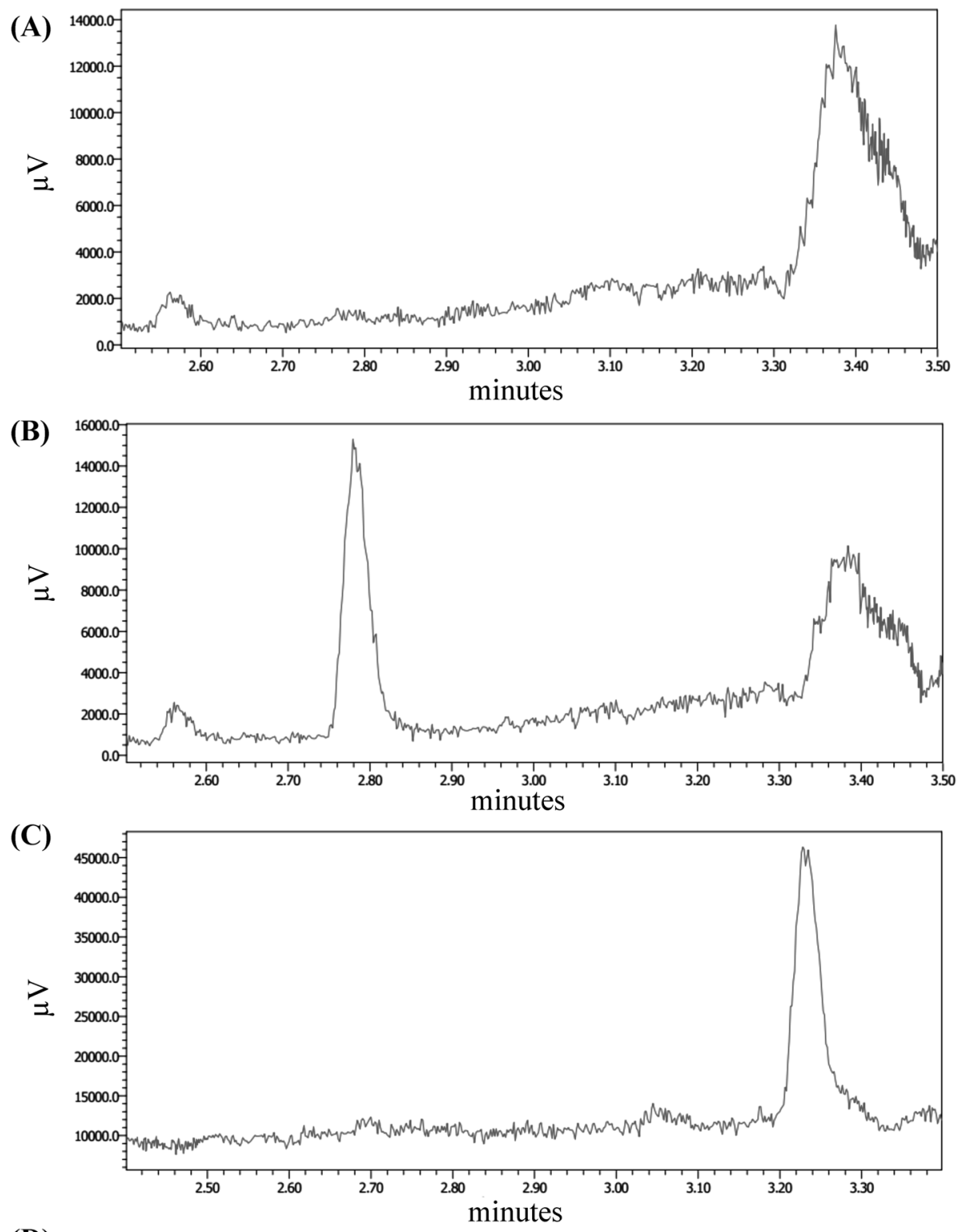

(D)

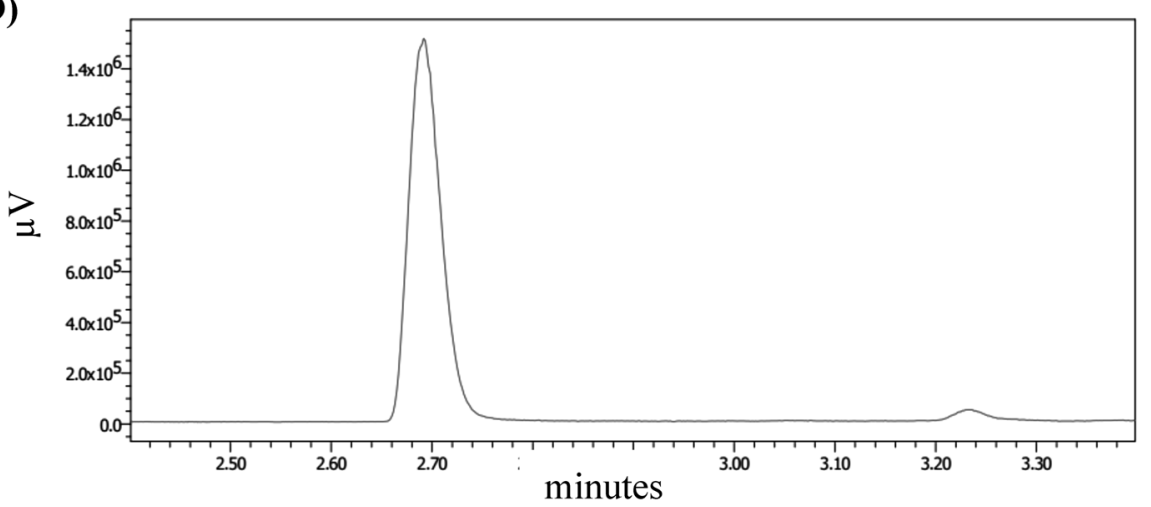

Fig. 2. Chromatograms of Letermovir and IS in Human Plasma; (A) Blank Plasma $m / z=573.30$; (B) Blank Plasma Spiked with Letermovir at LLOQ $(10 \mathrm{ng} / \mathrm{mL})$; (C) Blank Plasma $m / z=355.31$; (D) Blank Plasma Spiked with IS (500ng/mL) 
Table 2. Intra- and Inter-Assay Accuracy and Precision of Letermovir QC Samples

\begin{tabular}{|c|c|c|c|c|c|c|c|}
\hline \multirow[b]{2}{*}{ QC level } & \multirow{2}{*}{$\begin{array}{c}\text { Nominal } \\
\text { concentration } \\
(\mathrm{ng} / \mathrm{mL})\end{array}$} & \multicolumn{2}{|c|}{ Intra-assay $(n=5)^{b)}$} & \multicolumn{2}{|c|}{ Intra-assay $(n=5)^{c}$} & \multicolumn{2}{|c|}{ Inter-assay $(n=5)$} \\
\hline & & $\begin{array}{l}\text { Accuracy } \\
(\%)^{a)}\end{array}$ & $\begin{array}{c}\text { Precision } \\
(\text { RSD\%) }\end{array}$ & $\begin{array}{c}\text { Accuracy } \\
(\%)\end{array}$ & $\begin{array}{c}\text { Precision } \\
(\mathrm{RSD} \%)\end{array}$ & $\begin{array}{c}\text { Accuracy } \\
(\%)\end{array}$ & $\begin{array}{c}\text { Precision } \\
(\mathrm{RSD} \%)\end{array}$ \\
\hline LLOQ & 10 & 103 & 2.6 & 92 & 2.0 & 106 & 4.5 \\
\hline Low QC & 30 & 99 & 3.3 & 110 & 1.2 & 99 & 3.0 \\
\hline Mid QC & 500 & 101 & 1.9 & 108 & 0.4 & 100 & 3.6 \\
\hline High QC & 750 & 102 & 1.7 & 106 & 0.4 & 100 & 2.3 \\
\hline
\end{tabular}

LLOQ, lower limit of quantification; QC, quality control. a) Accuracy: (obtained concentration/nominal concentration) $\times 100, b$ ) Five individually prepared replicates, $c$ ) Five injections of one replicate.

Table 3. Matrix Effect $(n=6)$ and Recoveries of Letermovir and IS from Human Plasma $(n=6)$

\begin{tabular}{|c|c|c|c|c|c|c|}
\hline & \multirow[b]{2}{*}{ QC level } & \multirow{2}{*}{$\begin{array}{c}\text { Nominal } \\
\text { concentration } \\
(\mathrm{ng} / \mathrm{mL})\end{array}$} & \multicolumn{2}{|c|}{ Matrix effect } & \multicolumn{2}{|c|}{ Recovery } \\
\hline & & & $\begin{array}{l}\text { Absolute matrix } \\
\text { factor }^{a)}\end{array}$ & $\begin{array}{l}\text { Precision } \\
\left(\mathrm{RSD}^{\circ}\right)\end{array}$ & $\begin{array}{l}\text { Recovery } \\
(\%)^{b)}\end{array}$ & $\begin{array}{c}\text { Precision } \\
(\mathrm{RSD} \%)\end{array}$ \\
\hline \multirow[t]{3}{*}{ Letermovir } & Low QC & 30 & 1.5 & 8.5 & 93 & 8.0 \\
\hline & Mid QC & 500 & 1.3 & 4.0 & 97 & 4.8 \\
\hline & High QC & 750 & 1.3 & 3.3 & 94 & 4.9 \\
\hline Roscovitine (IS) & & 500 & 1.0 & 5.4 & 101 & 5.7 \\
\hline
\end{tabular}

QC, quality control. a) Absolute matrix factor: (peak area of analyte in human plasma/peak area of analyte in neat solvent). $b$ ) Recovery $\%$ : (peak area of analyte in prespiked plasma/peak area of analyte in post-spiked plasma) $\times 100$

\section{Selectivity}

The method showed good selectivity for letermovir and IS. The different chromatograms are presented in Fig. 2. Retention times of letermovir and IS were 2.78 and $2.69 \mathrm{~min}$, respectively. No endogenous peak was observed in the human plasma samples at the retention times of letermovir and IS.

\section{Sensitivity}

Sensitivity was evaluated with five LLOQ samples $(10 \mathrm{ng} / \mathrm{mL})$ as per the intra-assay accuracy and precision (Table 2). The results of the five replicates met the acceptance criteria of accuracy (nominal $\% \pm 15 \%$ ) and precision $(\mathrm{RSD} \%$ $\leq 15 \%)$. The LLOQ determined in this assay $(10 \mathrm{ng} / \mathrm{mL})$ was lower than the LLOQ established in a previous study by Isberner et al. $(100 \mathrm{ng} / \mathrm{mL}) .{ }^{14)}$

Accuracy, Precision, and Dilution Linearity

Table 2 summarizes the results of the intra-assay and interassay accuracy and precision. The accuracy of all the QCs at different levels was within $\pm 15 \%$, and precision was $\leq 15 \%$. Both the methods used in our study and in a previous study ${ }^{14}$ conformed with the FDA guidelines for bioanalytical method validation, in terms of accuracy and precision. ${ }^{15)}$ Dilution linearity with human blank plasma was investigated at a ratio of $1: 9$. The mean accuracy and precision values obtained were 103 and $0.5 \%$, respectively. All the results met the described acceptance criteria, suggesting that samples with a concentration $>1000 \mathrm{ng} / \mathrm{mL}$ can be quantified with good accuracy and precision.

\section{Matrix Effect and Recovery}

The results of the matrix effect and extraction recoveries of letermovir and IS from human plasma samples are presented in Table 3. The mean extraction recoveries and $\mathrm{RSD} \%$ at three levels of QCs were \pm 15 and $\leq 15 \%$, respectively, demonstrating a good recovery in different human plasma samples. The $\mathrm{RSD} \%$ value of the matrix effect expressing the inter-subject variability was $\leq 15 \%$. Furthermore, the RSD $\%$ of mean recovery and matrix effect of IS were also $\leq 15 \%$. These results suggest that simple protein precipitation with methanol, compared to liquid-liquid extraction used by Isberner et al., ${ }^{14)}$ is efficient for pre-treatment of the samples and contributes to optimized sample preparation time while achieving a good recovery during extraction.

Stability

The results of the stability studies are summarized in Tables 4 and 5. Letermovir stock solutions were stable for at least 1 year when prepared in a neat solvent. Furthermore, nominal\% of QCs at three concentration levels after $30 \mathrm{~d}$ at -20 and $-80^{\circ} \mathrm{C}$ were within $\pm 15 \%$. Thus, letermovir is stable for at least $30 \mathrm{~d}$ in human plasma under the described storage conditions.

\section{Conclusion}

A rapid, simple, and sensitive bioanalytical method for the quantification of letermovir in human plasma was developed and validated in accordance with FDA guidelines. ${ }^{15)}$ This method constitutes the first described LC/MS method for the quantification of letermovir in human plasma. The advantage of this method lies in its short analysis time of $6.1 \mathrm{~min}$ compared to $20 \mathrm{~min}$ in a previous HPLC assay with a retention time of $5.79 \pm 0.15$ min. $^{14)}$ Moreover, it has a simple method of sample preparation and high sensitivity following the use of a mass spectrometer detector through a single ion recording. The assay was fully validated with good selectivity and linearity over a large range of $10-1000 \mathrm{ng} / \mathrm{mL}$. Letermovir was stable in neat solvent and plasma for at least 1 year and $30 \mathrm{~d}$, respectively. A limitation of this study is the absence of clinical application on plasma samples of patients receiving letermovir treatment. Thus, an assessment of the assay using patients' samples is required.

In conclusion, the method was successfully used to quantify letermovir in human plasma. This work could be useful to perform not only clinical PK/PD studies but also to investigate drug-drug interactions and letermovir side effects, which 
Table 4. Stability Results of Letermovir in Human Plasma at Different Conditions $(n=5)$

\begin{tabular}{|c|c|c|c|}
\hline Stability condition & $\begin{array}{l}\text { Nominal } \\
\text { concentration } \\
(\mathrm{ng} / \mathrm{mL})\end{array}$ & $\begin{array}{l}\text { Nominal }^{a)} \\
\quad(\%)\end{array}$ & $\begin{array}{l}\text { Precision } \\
\text { (RSD\%) }\end{array}$ \\
\hline \multirow{3}{*}{$\begin{array}{l}\text { Auto sampler } \\
\qquad\left(10^{\circ} \mathrm{C}, 24 \mathrm{~h}\right)\end{array}$} & 30 & 106 & 2.3 \\
\hline & 500 & 104 & 2.2 \\
\hline & 750 & 103 & 1.4 \\
\hline Freeze-thaw & 30 & 101 & 4.4 \\
\hline \multirow{2}{*}{$\begin{array}{l}\text { Three cycles } \\
\qquad\left(-20^{\circ} \mathrm{C} / \text { r.t., } 24 \mathrm{~h}\right)\end{array}$} & 500 & 103 & 5.4 \\
\hline & 750 & 105 & 3.2 \\
\hline \multirow{3}{*}{$\begin{array}{l}\text { Post-preparation } \\
\qquad\left(-20^{\circ} \mathrm{C}, 48 \mathrm{~h}\right)\end{array}$} & 30 & 102 & 3.7 \\
\hline & 500 & 98 & 1.7 \\
\hline & 750 & 94 & 2.0 \\
\hline \multirow{3}{*}{$\begin{array}{l}\text { Room temperature } \\
24^{\circ} \mathrm{C}, 24 \mathrm{~h}\end{array}$} & 30 & 99 & 3.1 \\
\hline & 500 & 99 & 3.6 \\
\hline & 750 & 96 & 3.0 \\
\hline \multirow[t]{3}{*}{$-20^{\circ} \mathrm{C}, 24 \mathrm{~h}$} & 30 & 110 & 6.0 \\
\hline & 500 & 102 & 4.3 \\
\hline & 750 & 104 & 2.7 \\
\hline \multirow[t]{3}{*}{$-20^{\circ} \mathrm{C}, 10 \mathrm{~d}$} & 30 & 91 & 2.8 \\
\hline & 500 & 96 & 1.7 \\
\hline & 750 & 98 & 1.4 \\
\hline \multirow[t]{3}{*}{$-20^{\circ} \mathrm{C}, 30 \mathrm{~d}$} & 30 & 112 & 5.4 \\
\hline & 500 & 111 & 2.6 \\
\hline & 750 & 110 & 2.8 \\
\hline \multirow[t]{3}{*}{$-80^{\circ} \mathrm{C}, 30 \mathrm{~d}$} & 30 & 110 & 6.1 \\
\hline & 500 & 110 & 2.3 \\
\hline & 750 & 108 & 5.1 \\
\hline
\end{tabular}

a) Nominal $\%=($ response at the stability condition/response of freshly prepared sample) $\times 100$

Table 5. Stability Results of Letermovir Stock Solution at Different Conditions $(n=3)$

\begin{tabular}{lccc}
\hline \hline Stability condition & $\begin{array}{c}\text { Nominal } \\
\text { concentration } \\
(\mathrm{ng} / \mathrm{mL})\end{array}$ & $\begin{array}{c}\text { Nominal } \\
(\%)^{a)}\end{array}$ & $\begin{array}{c}\text { Precision } \\
(\mathrm{RSD} \%)\end{array}$ \\
\hline Room temperature, $6 \mathrm{~h}$ & 1000 & 97 & 4.1 \\
$-20^{\circ} \mathrm{C}, 6$ months & 1000 & 90 & 2.6 \\
$-20^{\circ} \mathrm{C}, 12$ months & 1000 & 87 & 0.9 \\
\hline
\end{tabular}

a) Nominal\% = (peak area of letermovir at stability condition/peak area of freshly prepared stock solution) $\times 100$

will be instructive in the creation of a dosage regimen and optimization of letermovir safety and efficiency.

Acknowledgments This work was supported by JSPS KAKENHI Grant Numbers: JP18K14951 to T. Yamada, and JK20K16078 to K. Suetsugu.
Conflict of Interest The authors declare no conflict of interest.

\section{References}

1) Chemaly R. F., Ullmann A. J., Stoelben S., Richard M. P., Bornhauser M., Groth C., Einsele H., Silverman M., Mullane K. M., Brown J., Nowak H., Kolling K., Stobernack H. P., Lischka P., Zimmermann H., Rubsamen-Schaeff H., Champlin R. E., Ehninger G., Team A. I. C. S., N. Engl. J. Med., 370, 1781-1789 (2014).

2) Goldner T., Hewlett G., Ettischer N., Ruebsamen-Schaeff H., Zimmermann H., Lischka P., J. Virol., 85, 10884-10893 (2011).

3) Marty F. M., Ljungman P., Chemaly R. F., et al., N. Engl. J. Med., 377, 2433-2444 (2017).

4) Schreiber A., Harter G., Schubert A., Bunjes D., Mertens T., Michel D., Expert Opin. Pharmacother., 10, 191-209 (2009).

5) Lischka P., Hewlett G., Wunberg T., Baumeister J., Paulsen D., Goldner T., Ruebsamen-Schaeff H., Zimmermann H., Antimicrob. Agents Chemother., 54, 1290-1297 (2010).

6) Prevymis $^{\mathrm{TM}}$ [Package insert]. Merck \& Co., Inc., Whitehouse Station, NJ.: 〈https://www.accessdata.fda.gov/drugsatfda_docs/label/ 2019/209939s005,209940s003lbl.pdf), cited 14 November, 2019.

7) Frietsch J. J., Michel D., Stamminger T., Hunstig F., Birndt S., Schnetzke U., Scholl S., Hochhaus A., Hilgendorf I., Mediterr. J. Hematol. Infect. Dis., 11, e2019001 (2019).

8) Jung S., Michel M., Stamminger T., Michel D., BMC Infect. Dis., 19, 388 (2019).

9) Komatsu T. E., Hodowanec A. C., Colberg-Poley A. M., Pikis A. Singer M. E., O'Rear J. J., Donaldson E. F., Antiviral Res., 169, 104549 (2019).

10) Kropeit D., von Richter O., Stobernack H. P., Rubsamen-Schaeff H., Zimmermann H., Clin. Pharmacol. Drug. Dev., 7, 9-21 (2018).

11) McCrea J. B., Macha S., Adedoyin A., Marshall W., Menzel K., Cho C. R., Liu F., Zhao T., Levine V., Kraft W. K., Yoon E., Panebianco D., Stoch S. A., Iwamoto M., J. Clin. Pharmacol., 59, 1331-1339 (2019).

12) Marshall W. L., McCrea J. B., Macha S., Menzel K., Liu F., van Schanke A., de Haes J. I. U., Hussaini A., Jordan H. R., Drexel M., Kantesaria B. S., Tsai C., Cho C. R., Hulskotte E. G. J., Butterton J. R., Iwamoto M., J. Clin. Pharmacol., 58, 897-904 (2018).

13) Kropeit D., Scheuenpflug J., Erb-Zohar K., Halabi A., Stobernack H. P., Hulskotte E. G. J., van Schanke A., Zimmermann H., RubsamenSchaeff H., Br. J. Clin. Pharmacol., 83, 1944-1953 (2017).

14) Isberner N., Aghai F., Schirmer D., Kraus S., Grigoleit G. U., Klinker H., J. Chromatogr. B Analyt. Technol. Biomed. Life Sci., 1159, 122399 (2020).

15) U.S. Department of Health and Human Services, Food and Drug Admnistration, Center for Drug Evaluation and Research, "Guidance for industry: Bioanalytical method validation." May 2018: 〈https://www.fda.gov/downloads/Drugs/GuidanceComplianceRegulatory Information/Guidances/ucm070107.pdf, cited 10 October, 2019.

16) Sakurai N., Nakamura Y., Kawagachi H., Abe J., Yamada K., Nagayama K., Kakeya H., Chem. Pharm. Bull., 67, 439-444 (2019).

17) Gu H., Liu G., Wang J., Aubry A. F., Arnold M. E., Anal. Chem., 86, 8959-8966 (2014). 